\title{
ON THE STRONG PARROTT COMPLETION PROBLEM
}

\author{
MIHÁLY BAKONYI AND HUGO J. WOERDEMAN
}

(Communicated by Palle E. T. Jorgensen)

\begin{abstract}
In this paper we parametrize all solutions of the Strong Parrott problem and obtain necessary and sufficient conditions for existence of isometric, coisometric, and unitary solutions.
\end{abstract}

\section{INTRODUCTION}

Let $\mathscr{H}_{1}, \mathscr{H}_{2}, \mathscr{K}_{1}$, and $\mathscr{K}_{2}$ be Hilbert spaces and

$$
\left(\begin{array}{cc}
B_{11} & B_{12} \\
? & B_{22}
\end{array}\right): \mathscr{H}_{1} \oplus \mathscr{H}_{2} \rightarrow \mathscr{K}_{1} \oplus \mathscr{K}_{2}
$$

be a partial matrix with $B_{11}, B_{12}$, and $B_{22}$ known (bounded) linear operators and $B_{21}$ unknown (indicated by “?”). Further, let also be given the Hilbert space $\mathscr{H}$ and the operators

$$
S=\left(\begin{array}{l}
S_{1} \\
S_{2}
\end{array}\right): \mathscr{H} \rightarrow \mathscr{H}_{1} \oplus \mathscr{H}_{2}, \quad T=\left(\begin{array}{c}
T_{1} \\
T_{2}
\end{array}\right): \mathscr{H} \rightarrow \mathscr{K}_{1} \oplus \mathscr{K}_{2} .
$$

We want to find the contractive completions of the problem

$$
\left(\begin{array}{cc}
B_{11} & B_{12} \\
? & B_{22}
\end{array}\right)\left(\begin{array}{l}
S_{1} \\
S_{2}
\end{array}\right)=\left(\begin{array}{c}
T_{1} \\
T_{2}
\end{array}\right)
$$

i.e., we want to find the contractions $B=\left(\begin{array}{ll}B_{11} & B_{12} \\ B_{21} & B_{22}\end{array}\right)$ such that $B S=T$. The special case $S^{*} S=T^{*} T$ called the Strong Parrott problem was considered by Foias and Tannenbaum in [5]. The Strong Parrott problem arose out of questions in the theory of intertwining dilations (see [4]).

For problem (1.3) we establish necessary and sufficient conditions for the existence of a contractive solution. For this purpose, based on an observation due to Timotin [7], we reduce the problem to a positive semidefinite completion problem. In the case when a solution exists, we obtain a parametrization for the

Received by the editors June 10, 1991.

1991 Mathematics Subject Classification. Primary 47A20; Secondary 47A65.

Key words and phrases. Strong Parrott problem, contractive completions, parametrization, (co-)isometric completions. 
set of all solutions as well as necessary and sufficient conditions for the existence of an isometric, coisometric, and unitary solution.

\section{THE RESULTS}

Theorem 2.1. Problem (1.3) has a contractive solution if and only if the following conditions are satisfied:

(i) $\left\|\left(B_{11} B_{12}\right)\right\| \leq 1$,

(ii) $B_{11} S_{1}+B_{12} S_{2}=T_{1}$,

(iii) the operator matrix

$$
\left(\begin{array}{cc}
I-B_{12}^{*} B_{12}-B_{22}^{*} B_{22} & S_{2}-B_{12}^{*} T_{1}-B_{22}^{*} T_{2} \\
S_{2}^{*}-T_{1}^{*} B_{12}-T_{2}^{*} B_{22} & S_{1}^{*} S_{1}+S_{2}^{*} S_{2}-T_{1}^{*} T_{1}-T_{2}^{*} T_{2}
\end{array}\right)
$$

is positive semidefinite.

Proof. Using a Schur complement argument, one easily sees that there exists a contractive solution of problem (1.3) if and only if there exists a positive semidefinite completion of the problem

$$
\left(\begin{array}{ccccc}
I & 0 & S_{1} & B_{11}^{*} & ? \\
0 & I & S_{2} & B_{12}^{*} & B_{22}^{*} \\
S_{1}^{*} & S_{2}^{*} & S^{*} S & T_{1}^{*} & T_{2}^{*} \\
B_{11} & B_{12} & T_{1} & I & 0 \\
? & B_{22} & T_{2} & 0 & I
\end{array}\right) .
$$

This was observed earlier by Timotin when $S^{*} S=T^{*} T$.

It is well known (see [2]) that the existence of a positive semidefinite completion of (2.2) is equivalent to the positive semidefiniteness of the two $4 \times 4$ principal submatrices of (2.2) formed with known entries. The positive semidefiniteness of the upper one can be reduced by a Schur complement argument to conditions (i) and (ii) while the positive semidefiniteness of the lower one is equivalent to (iii).

In the case when $S^{*} S=T^{*} T$, condition (iii) reduces to $\left\|\left(\begin{array}{l}B_{12} \\ B_{22}\end{array}\right)\right\| \leq 1$ and $S_{2}=B_{12}^{*} T_{1}+B_{22}^{*} T_{2}$. In this way, we recover the result of [5, Theorem 2] (see also [7]).

Before stating our parametrization, we introduce some notation and recall some results. For a linear operator $T$ we denote by $\overline{\mathscr{R}}(T)$ the closure of its range. For a contraction $T: \mathscr{F} \rightarrow \mathscr{G}$ we denote $D_{T}=\left(I-T^{*} T\right)^{1 / 2}$ and $\mathscr{D}_{T}=\overline{\mathscr{R}}\left(D_{T}\right)$.

Remark. Consider the Hilbert spaces $\mathscr{F}$ and $\mathscr{G}, \mathscr{F}$ a subspace of $\mathscr{F}$ and $T: \mathscr{F} \rightarrow \mathscr{G}$ a contraction. It is known that (see for instance [1]) there exists a one-to-one correspondence between the set of all contractions $T^{\prime}: \mathscr{F} \rightarrow \mathscr{G}$ with $T^{\prime} \mid \mathscr{F}_{0}=T$ and the set of all contractions $G: \mathscr{F} \ominus \mathscr{F}_{0} \rightarrow \mathscr{D}_{T^{*}}$ via

$$
T^{\prime}=\left(T D_{T^{*}} G\right): \mathscr{F}_{0} \oplus\left(\mathscr{F} \ominus \mathscr{F}_{0}\right) \rightarrow \mathscr{G} .
$$

Moreover, we have that

$$
\operatorname{dim} \mathscr{D}_{T^{\prime}}=\operatorname{dim} \mathscr{D}_{T} \oplus \mathscr{D}_{G}, \quad \operatorname{dim} \mathscr{D}_{T^{*}}=\operatorname{dim} \mathscr{D}_{G^{*}}
$$


Next consider matrix (1.1) with

$$
\left\|\left(B_{11} B_{12}\right)\right\| \leq 1, \quad\left\|\left(\begin{array}{l}
B_{12} \\
B_{22}
\end{array}\right)\right\| \leq 1 .
$$

The sufficiency of these two conditions for the existence of a contractive completion of (1.1) (i.e., without the additional requirement $B S=T$ ) was first proved in [6]. The contractivity of the operators in (2.5) imply by (2.3) the existence of contractions $G_{1}: \mathscr{H}_{1} \rightarrow \mathscr{D}_{B_{12}^{*}}$ and $G_{2}: \mathscr{D}_{B_{12}} \rightarrow \mathscr{K}_{2}$ such that

$$
B_{11}=D_{B_{12}^{*}} G_{1}, \quad B_{22}=G_{2} D_{B_{12}} .
$$

It was proved in $[1,3]$ that there exists a one-to-one correspondence between the set of all contractive completions of (1.1) and the set of all contractions $\Gamma: \mathscr{D}_{G_{1}} \rightarrow \mathscr{D}_{G_{2}^{*}}$ given by

$$
B_{21}=-G_{2} B_{12}^{*} G_{1}+D_{G_{2}^{*}} \Gamma D_{G_{1}} .
$$

Moreover, in [1] it was proved that if $B=\left(\begin{array}{ll}B_{11} & B_{12} \\ B_{21} & B_{22}\end{array}\right)$ is the completion corresponding to the parameter $\Gamma$ in (2.7) then

$$
\operatorname{dim} \mathscr{D}_{B}=\operatorname{dim} \mathscr{D}_{G_{2}} \oplus \mathscr{D}_{\Gamma}
$$

and

$$
\operatorname{dim} \mathscr{D}_{B^{*}}=\operatorname{dim} \mathscr{D}_{G_{1}^{*}} \oplus \mathscr{D}_{\Gamma^{*}}
$$

Let us return to problem (1.3) and assume that the conditions of Theorem 2.1 are satisfied, so the problem admits a contractive solution. Any solution of problem (1.3) is, in particular, a solution for the unconstrained problem (1.1) and, therefore, $B_{21}$ must be of form (2.7). The equation $B_{11} S_{1}+B_{12} S_{2}=T_{2}$ implies that if $\Gamma$ is the parameter in (2.7) of a solution $B$ then

$$
\Gamma \mid \overline{\mathscr{R}}\left(D_{G_{1}} S_{1}\right)=\Gamma_{0}
$$

where $\Gamma_{0}: \overline{\mathscr{R}}\left(D_{G_{1}} S_{1}\right) \rightarrow \mathscr{D}_{G_{2}^{*}}$ is uniquely determined by

$$
D_{G_{2}^{*}} \Gamma_{0} D_{G_{1}} S_{1}:=T_{2}-B_{22} S_{2}+G_{2} B_{12}^{*} G_{1} S_{1} .
$$

We are ready to state our parametrization results.

Theorem 2.2. Consider the linearly constrained completion problem (1.3) where the data satisfies the conditions of Theorem 2.1. Then there exists a one-to-one correspondence between the set of all contractive solutions of the problem and the set of all contractions

$$
G: \operatorname{ker}\left(S_{1}^{*} D_{G_{1}} \mid \mathscr{D}_{G_{1}}\right) \rightarrow \mathscr{D}_{\Gamma_{0}^{*}}
$$

where $G_{1}$ is given by (2.6) and $\Gamma_{0}$ is defined by (2.11).

Proof. Following the discussion preceding this theorem, there exists a one-toone correspondence between the set of all contractive solutions of problem (1.3) and the set of all contractive extensions of $\Gamma_{0}$ to $\mathscr{D}_{G_{1}}$. The contractivity of $\Gamma_{0}$ is ensured by the existence of a solution for problem (1.3). By the remark with the particular data $\mathscr{F}=\mathscr{D}_{G_{1}}, \mathscr{G}=\mathscr{D}_{G_{2}^{*}}, \mathscr{F}_{0}=\overline{\mathscr{R}}\left(D_{G_{1}} S_{1}\right), \mathscr{F} \ominus \mathscr{F}_{0}=$ $\operatorname{ker}\left(S_{1}^{*} D_{G_{1}} \mid \mathscr{D}_{G_{1}}\right)$, and $T_{0}=\Gamma_{0}$, we obtain a one-to-one correspondence between 
all contractive extensions of $\Gamma_{0}$ to $\mathscr{D}_{G_{1}}$ and all contractions of the form (2.12), and so our result follows.

Corollary 2.3. In the hypothesis of Theorem 2.2, the linearly constrained completion problem (1.3) has a unique solution if and only if $\mathscr{D}_{G_{1}} \subseteq \overline{\mathscr{R}}\left(S_{1}\right)$ or $\Gamma_{0}$ is a coisometry.

Proof. Following Theorem 2.2, there exists a unique contractive completion if and only if one of the spaces involved in (2.12) is the zero space. It is easy to see that $\operatorname{ker}\left(S_{1}^{*} D_{G_{1}} \mid \mathscr{D}_{G_{1}}\right)=\{0\}$ is equivalent with $\mathscr{D}_{G_{1}} \subseteq \overline{\mathscr{R}}\left(S_{1}\right)$ while $\mathscr{D}_{\Gamma_{0}^{*}}=\{0\}$ is equivalent to $\Gamma_{0}$ being a coisometry.

Theorem 2.4. Consider the linearly constrained completion problem (1.3) where the data satisfies the necessary and sufficient conditions of Theorem 2.1.

(i) Problem (1.3) has a coisometric solution $B$ if and only if $G_{1}$ is a coisometry and

$$
\operatorname{dim} \mathscr{D}_{\Gamma_{0}^{*}} \leq \operatorname{dim}\left(\operatorname{ker} G_{1} \cap \operatorname{ker} S_{1}^{*}\right) .
$$

(ii) Problem (1.3) has an isometric solution $B$ if and only if $G_{2}$ is an isometry, $S^{*} S=T^{*} T$, and $\operatorname{dim} \mathscr{D}_{G_{1}} \leq \operatorname{dim} \mathscr{D}_{G_{2}^{*}}$.

(iii) Problem (1.3) has a unitary solution $B$ if and only if $G_{1}^{*}$ and $G_{2}$ are isometries, $S^{*} S=T^{*} T$, and $\operatorname{dim} \mathscr{D}_{G_{1}}=\operatorname{dim} \mathscr{D}_{G_{2}^{*}}$.

Proof. Let $B=\left(B_{i j}\right)_{i, j=1}^{2}$ be an arbitrary contractive completion of problem (1.3) and let $G$ denote the corresponding parameter from Theorem 2.3. Combining the remark, Theorem 2.2, and the relations (2.4), (2.8), and (2.9), we obtain

$$
\operatorname{dim} \mathscr{D}_{B}=\operatorname{dim} \mathscr{D}_{G_{2}} \oplus \mathscr{D}_{\Gamma_{0}} \oplus \mathscr{D}_{G}
$$

and

$$
\operatorname{dim} \mathscr{D}_{B^{*}}=\operatorname{dim} \mathscr{D}_{G_{1}^{*}} \oplus \mathscr{D}_{G^{*}}
$$

By (2.15) problem (1.3) has a coisometric solution if and only if $G_{1}$ is a coisometry and there is a coisometry $G: \operatorname{ker}\left(S_{1}^{*} D_{G_{1}} \mid \mathscr{D}_{G_{1}}\right) \rightarrow \mathscr{D}_{\Gamma_{0}^{*}}$, i.e., $\operatorname{dim} \mathscr{D}_{\Gamma_{0}^{*}} \leq$ $\operatorname{dim}\left(\operatorname{ker} S_{1}^{*} D_{G_{1}} \mid \mathscr{D}_{G_{1}}\right)$. Since $G_{1}$ is a coisometry, $D_{G_{1}}$ is the projection onto the kernel of $G_{1}$, so part (i) follows.

It is clear that the conditions $G_{2}$ is an isometry and $S^{*} S=T^{*} T$ are necessary for the existence of an isometric solution. Now, assuming that these two conditions are satisfied, if $B$ is a contractive solution of problem (1.3) corresponding to the parameter $\Gamma$ in $(2.7)$ satisfying $(2.10)$, then we have that

$$
S^{*}\left(I-B^{*} B\right) S=S^{*} S-T^{*} T=0 .
$$

A straightforward computation shows that in this case

$$
I-B^{*} B=\left(\begin{array}{cc}
D_{G_{1}} D_{\Gamma}^{2} D_{G_{1}} & 0 \\
0 & 0
\end{array}\right)
$$

and thus $D_{\Gamma} D_{G_{1}} S_{1}=0$. This latter relation implies that $\Gamma$ is an isometry on $\overline{\mathscr{R}}\left(D_{G_{1}} S_{1}\right)$, and thus (2.10) implies that $\Gamma_{0}$ is an isometry. Relation (2.14) implies that the existence of an isometric completion is equivalent with the conditions that $G_{2}$ and $\Gamma_{0}$ are isometric and there exists an isometry $G: \operatorname{ker}\left(S_{1}^{*} D_{G_{1}} \mid \mathscr{D}_{G_{1}}\right) \rightarrow \mathscr{D}_{\Gamma_{0}^{*}}$. So it remains to prove that $\operatorname{dim} \mathscr{D}_{\Gamma_{0}^{*}} \leq$ 
$\operatorname{dim}\left(\operatorname{ker} S_{1}^{*} D_{G_{1}} \mid \mathscr{D}_{G_{1}}\right)$ is equivalent with $\operatorname{dim} \mathscr{D}_{G_{1}} \leq \operatorname{dim} \mathscr{D}_{G_{2}^{*}}$. Since $\Gamma_{0}$ is an isometry, $\operatorname{dim} \mathscr{D}_{\Gamma_{0}^{*}}=\operatorname{dim} \mathscr{D}_{G_{2}^{*}}-\operatorname{dim} \overline{\mathscr{R}}\left(D_{G_{1}} S_{1}\right)$. The latter equality together with

$$
\operatorname{dim} \operatorname{ker}\left(S_{1}^{*} D_{G_{1}} \mid \mathscr{D}_{G_{1}}\right)=\operatorname{dim} \mathscr{D}_{G_{1}}-\operatorname{dim} \overline{\mathscr{R}}\left(D_{G_{1}} S_{1}\right)
$$

imply that $\operatorname{dim} \operatorname{ker}\left(S_{1}^{*} D_{G_{1}} \mid \mathscr{D}_{G_{1}}\right) \leq \operatorname{dim} \mathscr{D}_{\Gamma_{0}^{*}}$ is equivalent with $\operatorname{dim} \mathscr{D}_{G_{1}} \leq$ $\operatorname{dim} \mathscr{D}_{G_{2}}^{*}$ and (ii) follows.

The proof of (iii) is similar to that of (ii), but we must add the condition that $G_{1}$ is a coisometry and that there exists a unitary $G: \operatorname{ker}\left(S_{1}^{*} D_{G_{1}} \mid \mathscr{D}_{G_{1}}\right) \rightarrow \mathscr{D}_{\Gamma_{0}^{*}}$, which finally gives the condition $\operatorname{dim} \mathscr{D}_{G_{1}}=\operatorname{dim} \mathscr{D}_{G_{2}^{*}}$.

\section{REFERENCES}

1. Gr. Arsene and A. Gheondea, Completing matrix contractions, J. Operator Theory 7 (1982), 179-189.

2. H. Dym and I. Gohberg, Extensions of band matrices with band inverses, Linear Algebra Appl. 36 (1981), 1-24.

3. C. Davis, W. M. Kahan, and H. F. Weinberger, Norm preserving dilations and their applications to optimal error bounds, SIAM J. Numer. Anal. 19 (1982), 444-469.

4. C. Foias and A. E. Frazho, The commutant lifting approach to interpolation problems, Oper. Theory: Adv. Appl., vol. 44, Birkhäuser, Basel and Boston, 1990.

5. C. Foiaş and A. Tannenbaum, A Strong Parrott theorem, Proc. Amer. Math. Soc. 106 (1989), 777-784.

6. S. Parrott, On the quotient norm and the Sz.-Nagy-Foias Lifting theorem, J. Funct. Anal. 30 (1978), 311-328.

7. D. Timotin, A note on Parrott's Strong theorem, preprint.

Department of Mathematics, The College of William and Mary, Williamsburg, VIRGINIA 23187-8795

E-mail address, H. J. Woerdeman: hugo@cs.wm.edu 